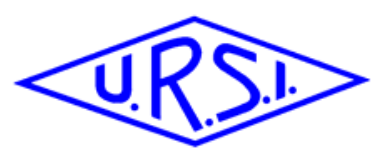

\title{
A new approach of V2X communications for Long Range Applications in UAVs
}

\author{
J.A. Godoy, F. Cabrera, V. Araña, D. Sánchez, I. Alonso and N. Molina \\ IDeTIC, Universidad de Las Palmas de Gran Canaria, http://www.ulpgc.es
}

\begin{abstract}
The importance of UAVs (Unmanned Aerial Vehicle), also called drones, in the society has been increasing over last years. Today, UAVs are used in different situations, for example on emergency conditions for locating people after an earthquake or transporting medicines to not accessible places.
\end{abstract}

A major drawback of drone's communications is the short distance that can be reached. In this paper, the perform of LoRa (Long-Range) protocol is studied with the purpose of implementing it in these kinds of communications. Also, a system is proposed with the goal of increasing coverage area in UAVs communications.

Furthermore, some tests were developed equipping two UAVs with the necessary hardware and transmitting information between two far points to analyze the performance of the system developed. Some of these values were the distance, power of transmission, the Signal to Noise ratio (SNR) and the Received Signal Strength Indicator (RSSI). Finally, the developed system was tested and gave a successful result for a distance of $10 \mathrm{~km}$.

\section{Introduction}

The impulse in the development of applications using UAVs (Unmanned Aerial Vehicles) has suffered a great increasing in recent years. UAVs can be used in different extreme situations, for example to locate people after a natural disaster, transport medicines to not easy accessible places or even in wildfire tracking applications [1].

According to this last application, wildfire tracking, it is necessary for monitoring large areas of natural tropical forest. The main objective of this is to detect possible fire hotspots early to prevent the fire advance.

Nevertheless, UAVs present a great disadvantage the short range of communication to a ground station and with other drones. So we consider necessary to implement a system that solve this problem.
Numerous papers have focused on evaluating the performance of different systems in order to increase the range of coverage. [2] uses an OFDM technique based on multi-carrier transmission technology. In [3] is proposed an adaptive vertical array antenna technique into WiBro (Wide Broadband) modem for UAV communication system. A collection of UAVs that communicate through a wireless link as a mobile ad-hoc network (MANET) using directional antenna is considered by [4].

In this paper, with the purpose of increase coverage area of two UAVs, a study of different wireless protocol has been made in order to determine which of these is the best protocol that can suit to the communications needs. The candidate protocols to be implemented in this system were: LoRa (Long Range) [5], IEEE 802.11p [6], SigFox [7], Bluetooth [8] and Zigbee [9].

Once the comparison of the different protocols has been made, it is decided that the protocol which best meets expectations is LoRa. So, in this paper we present a new approach of V2X communications for Long Range Applications in UAVs based on LoRa (Long Range). To the best of our knowledge, our research is the first to use LoRa for UAV communications.

The paper is organized as follows: Section 2 describes the LoRa protocol. Next, in Section 3, the proposed system based on LoRa is explained. In Section 4 and 5, the test environment and experimental results are described, respectively. Finally, Section 6 contains the conclusion of this paper, and future works are presented.

\section{LoRa protocol}

LoRa is a Long Range protocol designed for building IoT networks [5]. This protocol allows transmit low data rate information between two separated points over an approximate distance of 18 kilometers with a transmitted power of $25 \mathrm{~mW}(\approx 14 \mathrm{dBm})$ and approximately 9 kilometers with $5 \mathrm{~mW}(\approx 9,5 \mathrm{dBm})$. It was specifically devised to create low power consumption tracking applications [10]. Thus, parameters of different devices can be transmitted for monitoring the proper performance of these. 
The over-the-air modulation method that LoRa employs is a type of spread spectrum (DSSS [11], Direct Sequence Spread Spectrum) called CSS (Chirp Spread Spectrum). In this modulation each information bit is widened by a chipping factor (Figure 1). The number of chips assigned to each bit is called Spread Factor [12]. Furthermore, LoRa shifts the modulation through the channel, Frequency Hopping.

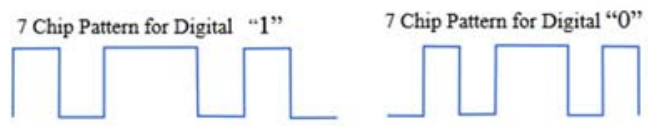

Data to Send over the air. Example is 1001

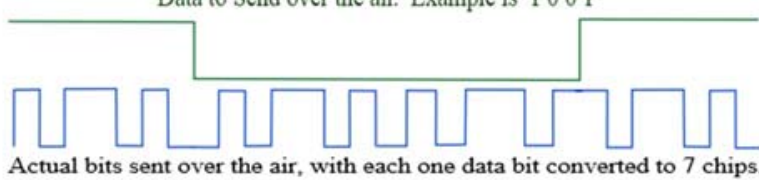

Figure 1. Spread Spectrum using chirps modulation example. Each information bit ( 0 or 1$)$ is replaced by a bit sequence called chirp.

LoRa works in the $868 \mathrm{MHz}$ (Europe) or $915 \mathrm{MHz}$ (USA) licensed bands and the channel bandwidth can be selected between $125 \mathrm{Khz}, 250 \mathrm{KHz}$ or $500 \mathrm{KHz}$ [13].

The minimum level of signal that LoRa can detect (sensitivity) is $-128 \mathrm{dBm}$, so the dynamic range between minimum transmitted power and sensitivity is around 137 dBm. [13]

LoRa offers different data rates in function of the channel bandwidth selected. The maximum data rate is around 300 kbps using a channel bandwidth of $500 \mathrm{KHz}$.

\section{Implemented system}

With the purpose of increasing the communication coverage using LoRa, the design of a low cost LoRa communications system adapted to drone communications is proposed (as presented in Figure 2).

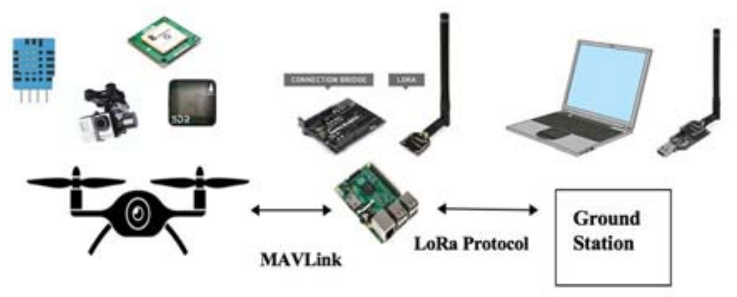

Figure 2. Designed system. It is formed by a UAV that send different parameters or information, a system which translate MAVLink messages into LoRa messages and a Ground Station that receive the information.

The system is conformed by an UAV that can be equipped with different sensors, for example a thermometer, a GPS, a camera, a Magnetometer, etc.
This information is sent by the UAV using MAVLink protocol [14] to a translator system which converts MAVLink messages into LoRa messages and vice versa. This device could be a microcontroller, for example a Raspberry Pi, equipped on board.

MAVLink (Micro Air Vehicle Communication Protocol) helps extract flight parameters from drones.

The LoRa transceiver used is the Semtech SX1272 [15] module designed by Libelium (see figure 3 ).

SX1272 transceiver is one of the most characteristic modems which implement LoRa protocol. It offers spread spectrum communications getting great link lengths and a good immunity performance against interference. Thereby, it makes the sensitivity level low enough to allow weak signals can be detected.

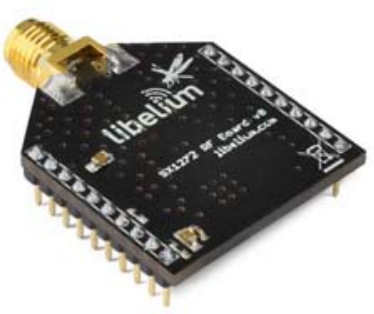

Figure 3. Semtech SX1272 module transceiver designed by Libelium company use in this prototype.

Main features of SX1272 LoRa transceiver are:

- High Sensitivity: a maximum of $-137 \mathrm{dBm}$.

- $\quad+14 \mathrm{dBm}$ Maximum transmitted power.

- $89 \mathrm{~dB}$ of blocking immunity.

- Accepts different modulation types: FSK, GFSK, MSK, GMSK, LoRa and OOK.

- Data rates of $300 \mathrm{kbps}$.

- Forward Error Correction.

- Preamble detection.

Also, SX1272 implements the LoRa European or USA standard in the same module. Mains difference between these standards are shown in table 1.

\begin{tabular}{|c|c|c|c|}
\hline $\begin{array}{c}\text { Frequency } \\
\text { bands }\end{array}$ & $\begin{array}{c}\text { Maximum } \\
\text { power of } \\
\text { transmission }\end{array}$ & Sensitivity & $\begin{array}{c}\text { Channel } \\
\text { numbers }\end{array}$ \\
\cline { 1 - 1 } $\begin{array}{c}863-870 \\
\mathrm{MHz} \\
\text { (Europe) }\end{array}$ & $14 \mathrm{dBm}$ & $-134 \mathrm{dBm}$ & 8 \\
\cline { 1 - 1 } $\begin{array}{c}902-938 \\
\mathrm{MHz} \\
\text { (USA) }\end{array}$ & & & 13 \\
\hline
\end{tabular}

Table 1. Differences Semtech sx1272 module transceiver features from Europe and USA. 
The ground station is formed by other SX1272 transceiver connected to a computer through to a Waspmote Gateway, also developed by Cooking Hacks (see figure 4).

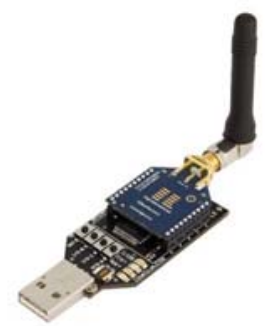

Figure 4. Waspmote Gateway designed by Cooking Hacks.

A software is run in order to receive the LoRa message with the information sent by the UAV, decode it and obtain the SNR and RSSI values to check the link status. This program also sent commands to the UAV, principally, send messages for requesting monitoring parameters to the UAV.

\section{Test}

The system was tested in different situations in order to check its correct functioning and determine the range coverage. In this experiment various radio links were made between different points of Gran Canaria Island, Spain (see figure 5).

Two UAVs were equipped with the necessary hardware and 100 bytes length packets were periodically sent.

In each radio link the transmitter was situated in the point named as "Teleco" and transmissions in different channels with different transmitted powers were used (0 $\mathrm{dBm}, 7 \mathrm{dBm}$ or $14 \mathrm{dBm})$. In the receiver, two important parameters were analyzed: Signal to Noise Rate (SNR) and Received Signal Strength Indicator (RSSI).

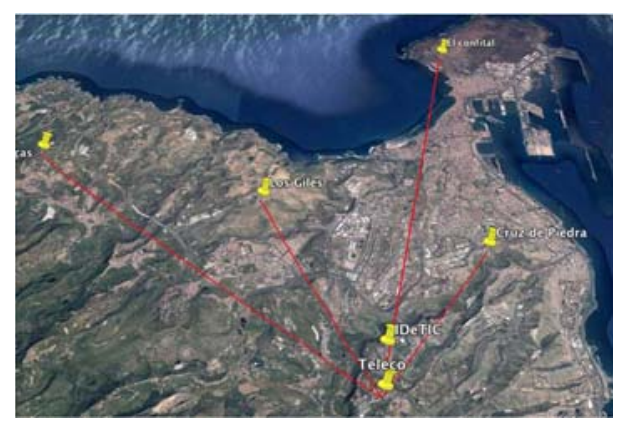

Figure 5. Image of diferents radio links implemented in the test fase.

In the Table 2, the length of each link is showed.

\begin{tabular}{|c|c|c|}
\hline Transmitter & Receiver & $\begin{array}{c}\text { Distance } \\
\text { (Kms) }\end{array}$ \\
\hline \multirow{4}{*}{ Teleco } & IDeTic & 0,96 \\
\cline { 2 - 3 } & $\begin{array}{c}\text { Cruz de } \\
\text { Piedra }\end{array}$ & 4 \\
\cline { 2 - 3 } & Los Giles & 5,1 \\
\cline { 2 - 3 } & Arucas & 9,04 \\
\cline { 2 - 3 } & El Confital & 10,19 \\
\hline
\end{tabular}

Table 2. Distance between transmitter and receiver in each radio link test.

\section{Results}

All radio links offers different information, but in general terms, all of them present good results, even better that we expected.

However, the radio link with the best results was using the Receiver in "El Confital" and, for this reason, we analyze these results here. However, results in each radio link were so good.

In figure 6 it shows the ground plane of this radiolink, also you can observe how there is direct vision between the transmitting equipment and the receiver. This is not really true, because this radio link is over Las Palmas de Gran Canaria city, so in the image is not represented the buildings and other objects in movements.

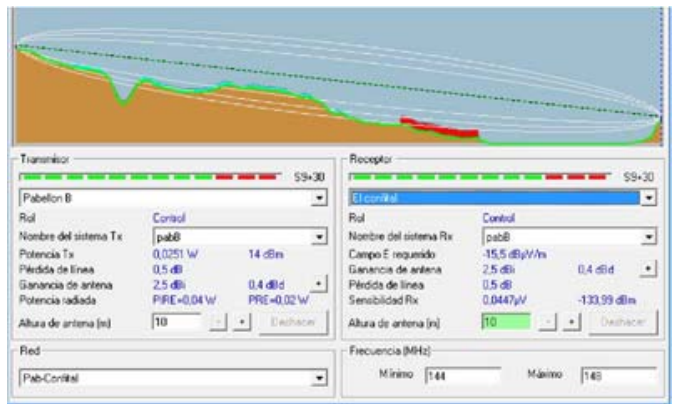

Figure 6. Ground plane calculated by Radio mobile application.

A communication range of 10,19 kilometers was reached with a power of transmitted signal of $0 \mathrm{dBm}$. This test was developed in an urban environment (across Las Palmas de Gran Canaria city) and with line of sight between the transmitter and the receiver. One important aspect of these results is that the environment is not the ideal, because in urban environments reflections and fading phenomena may appear more frequently.

For this reason, LoRa was selected because it is an idea protocol for IoT, this have a good response in this kind of communications. 
In table Table 3 is shown different results in function of power transmitted.

\begin{tabular}{|c|c|c|c|}
\hline $\begin{array}{c}\text { Power } \\
\text { transmitted } \\
(\mathbf{d B m})\end{array}$ & $\begin{array}{c}\text { Sensitivity } \\
\mathbf{( d B m})\end{array}$ & $\begin{array}{c}\text { SNR } \\
\mathbf{( d B m})\end{array}$ & $\begin{array}{c}\text { RSSI } \\
\mathbf{( d B m})\end{array}$ \\
\cline { 1 - 2 } 0 & \multirow{2}{*}{-134} & -5 & -122 \\
\cline { 1 - 1 } \cline { 3 - 4 } & & 0 & -118 \\
\hline 14 & & 3 & -106 \\
\hline
\end{tabular}

Table 3. RSSI and SNR values of received signal in function of Power transmitted.

As can be seen, transmitting a signal using LoRa modulation with a power level of $0 \mathrm{dBm}$ a dynamic margin of $12 \mathrm{~dB}$ is obtained.

\section{Conclusion}

As conclusion, LoRa offers good performance for long distance communications enough to transmit a UAV monitoring data such as position, battery status, temperature, etcetera.

The experiments carried out have endorsed that the proposed system meets the requirement of reaching a large communication distance, $10 \mathrm{kms}$ using a transmitted power of $0 \mathrm{dBm}$. However, according to results obtained in Table 3, the area of coverage can be increased due to with a transmitted power of $0 \mathrm{dBm}$ a dynamic margin of $12 \mathrm{~dB}$ is obtained.

\section{Acknowledgements}

The authors acknowledge the work carried out by Juan Domingo Santana Urbín for their enormous contribution during this research. This work was supported by the Spanish Government under Grant TEC2014-60283-C3-2$\mathrm{R}$ Project and the University of Las Palmas de Gran Canaria under Grant ULPGC2015-01 Project.

\section{References}

1. Ministerio de Agricultura, Alimentación y Medio Ambiente (2015). "Desarrollo de nuevas técnicas y Seguimiento de Líneas de Fuego en Incendios Forestales basados en sensors térmicos móviles de despliegue rápido (SeLiF)(Ref.686/2012).

2. Zhiqiang W., Kumar, H. \&Davari A. (2005) "Performance evaluation of OFDM transmission in UAV wireless communication," Proceedings of the ThirtySeventh Southeastern Symposium on System Theory, 2005. SSST '05., 2005, pp. 6-10.

3. Park, P. J., Choi , S. M., Dong-Hee, L. \& Byung-Seub, L. (2009 "Performance of UAV(Unmanned Aerial
Vehicle) communication system adapting WiBro with array antenna," 11th International Conference on Advanced Communication Technology, Phoenix Park, 2009, pp. 1233-1237.

4. Alshbatat, A. I. \& Dong, L. (2010) "Adaptive MAC protocol for UAV communication networks using directional antennas," 2010 International Conference on Networking, Sensing and Control (ICNSC), Chicago, IL, 2010, pp. 598-603.

5. LoRa Alliance. Wide Area Network for IOT. Recovered of: https://www.lora-alliance.org

6. Praksh, U., Pal, R. \& Gupta, N. (2015). Performance Evaluation of IEEE 802.11 p by Varying Data Rate and Node Density in Vehicular ad Hoc Nerwork.

7. "About SIGFOX”, Sigfox, [online] Available in: http://www.sigfox.com/en/\#!/about.

8. E. Ferro and F. Potorti. Bluetooth and wi-fi wireless protocols: a survey and a comparison. Wireless Communications, IEEE, 12(1):12-26, Feb. 2005.

9. ZigBee Specification 05347r17, January 2008 , available in Zigbee Alliance, www.zigee.org

10. Mikhaylov, K., Petäjäjärvi, J. \& Hänninen, T. (2016). Analysis of Capacity and Scalability of the LoRa Low Power Wide Area Network Technology. Centre for Wireless Communications. University of Oulu.

11. Waghmare, P. \& Nawkhare, R. (2015). Performance Evaluation of DSSS and FHSS Using Modulation Technique. International Journal of Innovative Research in Computer and Communication Engineering, Vol. 3, Issue 10 , October.

12.Semtech Corporation. (2015). LoRa Modulation Basics. AN1200.22, Revision 2.

13. Ruano, E., Tourancheau, B. \& Alphand, O. (2016). LoRa ${ }^{\mathrm{TM}}$ protocol Evaluations, Limitations and Practical Test. Univeristat Politecnica de Catalunya.

14. Oficial MavLink Web. Recovery in 26 January of 2017: http://qgroundcontrol.org/mavlink/start

15."SX1272/3/6/7/8: LoRa Modem Designer's Guide" Semtech Co., Camarillo, CA, AN1200.13, 2013. 